\title{
Citoyenneté et valeurs dans l’Eurafrique
}

\author{
Charles-Romain Mbele (Université d' Yaoundé I)
}

Résumé. Tenter de cerner la question de la «citoyenneté et des valeurs » avec quelque complexité et profondeur dépasse le face-à-face entre l'Europe et l'Afrique. D'une part, dans une série de conférences à l'aube du 21e siècle, l'Unesco s'est demandé «Où vont les valeurs ? » D'autre part, « les liens préférentiels » entre l'Europe et l'Afrique sont désormais sous la juridiction de l'Organisation mondiale du Commerce. De ce point de vue, interroger le statut politique et civique des hommes et des femmes dans le cadre institutionnel et partenarial de l'Eurafrique, c'est en creux se demander quel sort est réservé, par l'économie-monde actuelle, au fait d'être citoyen. Étant donné le déséquilibre qui caractérise l'Eurafrique, ce n'est qu'avec l'engagement actif des citoyens qui votent et participent à une société civile critique qu'un nouveau partenariat favorable aux Africains pourrait s'actualiser.

\begin{abstract}
Attempting to plumb the complexity and depth of the issue of citizenship and values goes well beyond the interface between Europe and Africa. On one hand, in a series of communications at the beginning of the 21st century, UNESCO asked itself, "Where are the values?" On the other hand, the "preferential links" between Europe and Africa are henceforth under the jurisdiction of the World Trade Organization. From this point of view, questioning the political and civic status of men and women in the institutional framework and partnership of Eurafrica, means to ask oneself what destiny is reserved, by the current world economy, to the fact of being citizen. Given the disequilibrium that characterizes Eurafrica, it is only with the active engagement of citizens who vote and participate in a critical civil society that a new partnership favorable to Africans could be actualized.
\end{abstract}

\section{Être en commun : Europe et Afrique}

Doit-on interroger, du point de vue de la pensée critique, les «liens » entre l'Europe et l'Afrique? En Afrique subsaharienne, malgré un réel intérêt pour l'actualité historique, la philosophie morale, politique et du droit a laissé un tel questionnement à l'expertise spécialisée en économie, en politologie et à une diplomatie sans dessein d'envergure. Sans hésiter, la pensée africaine d'hier notamment avec Kwamé Nkrumah, Frantz Fanon, Sally N'dongo, Marcien Towa, etc.-a réfléchi sur de telles questions (voir par exemple, Diop, 1960). Le silence assourdissant actuel peut se faire complice d'un dessein politique qui nourrit un double impensé philosophique. D'une part il y a la distinction du double statut politique naguère théorisé dans la citoyenneté différenciée (citoyens/non-citoyens, hommes/sous-hommes, civilisés/barbares) et d'autre part, une dogmatique rédemptrice, messianique et bienveillante caractérisée par 
la tendance à faire le bien des Africains malgré eux, tout en corsetant leur parole libre. Aussi ne sont-ils jamais consultés sur l'essentiel, notamment en donnant leur avis, par le vote, sur les accords qui lient l'Afrique à l'Europe. Or, dans ces accords qui associent l'Afrique subsaharienne à Bruxelles, est en jeu la question de l'être-en-commun, essentiellement le statut politique d'hommes, de femmes et d'enfants dont le rapport de leur liberté avec elle-même et avec le monde des choses est affaibli par les «fruits empoisonnés» (Chossudovsky, 1994) d'un ajustement économique visant à intégrer au forceps le continent africain à l'économie mondiale (Braeckman, 2003). Comprendre théoriquement cet ébranlement est déjà une volonté de le dépasser et de lui opposer une alternative africaine, comme pensée de la citoyenneté postulant qu'est possible un être et un vivre-ensemble hors de l'exploitation et de l'exclusion.

\section{Valeurs : Délégitimation ou universalité?}

Parler des valeurs aujourd'hui pose un problème culturel. On parle de «pensée faible ", d' "ontologie faible ", de refus de "valeurs centrales", de délégitimation des "grands récits », d'absence de critères du jugement moral. Le relativisme propose le repli sur ses valeurs culturelles et religieuses propres. Pour le pragmatisme relativiste et postmoderne et pour le culturalisme qui ontologisent les civilisations, il n'y a pas d' "universaux de l'anthropos ». Penser l'universalité des valeurs (universalisation réelle des droits de l'homme, de la démocratie et de la techno-science), c'est être un fauteur de guerre dans un monde subdivisé en ami/ennemi, en civilisés/barbares ou « voyous ».

Les valeurs des Lumières (la raison, le progrès historique et culturel, l'égalité entre les hommes), de l'État-nation (son indépendance et sa souveraineté, sa culture, son système éducatif, son économie, son histoire, son indépendance alimentaire et énergétique), du développement (entendu comme la maîtrise de la civilisation industrielle) sont désormais de fausses valeurs. Le primat de l'identité et la dialectique de la libération des peuples voulant accéder au processus d'universalisation de la civilisation industrielle sont rejetés par l'éthos globalitaire. La tendance du primat de l'identité, en refusant de séparer libération économique et politique et libération culturelle, exprime une certaine défense de l'originalité et de la valeur des cultures nationales; elle est accusée de nativisme archaïque. La dialectique de la libération qui veut prendre en compte les affrontements et les oppositions du point de vue conceptuel que socio-politique, est rejetée comme un radicalisme. En effet, l'éthos globaliste veut un sujet sans frontières. Ce nouveau sujet doit être «cosmopolitique », « hybride », « nomade », « métis », parce que l'humus culturel devant nourrir le commerce et le consumérisme se satisfait d'un consommateur sans attaches, « sans ancrages », « un soi déterritorialisé » aux « ancestralités multiples ». Il n'y a plus désormais, selon l'idéologie mondialiste que relaie l'irénisme de la pensée africaine récente, d'affrontements verbaux, conceptuels, sociaux et politiques, le monde n'étant qu'une suite d'opportunités. Flexible, désocialisé, le 
sujet sans frontières doit « fluctuer d'une personnalité ou d'un rôle à un autre au gré des rencontres, des unions, des mélanges, des besoins, des usages ou des utilités, sans qu'il y ait là aucun ordre, aucun axe, aucun nom privilégié » (Balibar, 2001, p.117). Dès lors, penser les fondements et les fins, c'est spéculer sur le désirable ou l'idéal, et c'est par conséquent basculer dans l' " irrationnel ». Il s'agit seulement de valoriser l'excroissance infinie de la multiplicité, le passage continu et réversible d'une identité à une autre, la mobilité et le flux perpétuel pour lesquels on est tout et rien. Est ainsi conceptualisée la fin des liens dans un monde qui n'en offre plus. Cette vision du monde prend acte d'un état pour le renforcer métaphysiquement. Malgré la fiction «globalitaire », le soi déterritorialisé reste arc-bouté sur la problématique de la propriété et des seuls droits individuels formels, là où le grand nombre n'a accès à rien.

\section{Perspectives africaines}

Le point de vue adopté ici s'oppose donc aussi à d'autres aspects de l'idéologie mondialiste dont la philosophie partenariale n'est qu'un aspect: la fragmentation du sujet postmoderne, la hiérarchisation des hommes lors de la bataille inaugurale pour la megalothymia fantasmée par l'Américain Francis Fukuyama dans La Fin de l'histoire. Une perspective africaine sur le temps long de la citoyenneté fait voir que l'universel existe, non comme une idée platonicienne, mais comme lutte pour que l'idéal postulé (la liberté, l'égalité, l'amitié, les droits de l'homme, etc.) s'incarnent dans des pratiques normatives des formes culturelles et historiques concrètes, particulières. Plus que jamais peut-être, il se constitue, de façon inchoative, dans l'ordre éthique idéal porté par les Conférences internationales, une conscience des valeurs communes (environnement, femme, développement, justice internationale). Réaliser cette nouvelle cité humaine exige la conceptualisation des valeurs universelles qui donnent des limites au marché pour que d'autres forces s'approprient politiquement l'économie. Le contractualisme ultralibéral, en privilégiant le contrat par rapport à la loi, laisse irreprésentables les intérêts incapables d'organisation politique, notamment ceux des peuples du Sud, des exclus (chômeurs, sans domicile fixe, jeunes désespérés des banlieues, immigrés non nationaux). C'est, les yeux fixés sur cet horizon, que nous voulons penser le sujet politique dans l'espace public où se nouent les liens institutionnels de l'Europe et l'Afrique. D'abord il y a les anciens liens qui datent du Traité de Rome (1957) : la politique de l'aide qui en était le centre s'est matérialisée sous une forme conventionnelle au moyen des conventions de Yaoundé et de Lomé (Pierre Jalée, 1973). Il y a ensuite ceux qui unissent ces deux mondes après la bipolarité : elle a été formalisée autour d'une philosophie partenariale («accords de partenariat», «nouveau partenariat»). Mais une même logique messianique les parcoure, celle qui consiste à vouloir faire le bien des Africains sans les consulter, malgré eux. Il s'est ajouté la volonté d'inscrire une destination qui se pare des atours de la nature dont l'étape nécessaire et incontournable serait 
l'«accumulation primitive du capital» (New Partnership for Africa's Development, 2001).

\section{Conception géopolitique de l'Eurafrique et question de l'éducation}

Un exposé historique-critique s'impose ici pour mettre cette volonté en perspective. L'ancien et le nouveau partenariat tournent autour du vieux concept géopolitique de l'Eurafrique. Les milieux d'affaires européens voulaient sceller une "relation privilégiée » entre les puissances coloniales (France, Belgique, Angleterre) et leurs colonies pour faire contrepoids aux États-Unis et à l'Union soviétique. Le but : accomplir le progrès et la renaissance de l'Europe. Ce fut d'abord la politique mercantiliste du "pacte colonial» dans les années $30 \mathrm{du}$ 20e siècle. Avec la naissance du Marché Commun à la fin des années 50, et celle, concomitante, de l'Association des États africains et malgaches, la politique menée est celle de l'assistance technique. Le "nouveau partenariat» actuel entre l'Union européenne et l'Afrique, les Caraïbes et le Pacifique n'en est qu'une continuité : il tourne, formellement, comme les politiques précédentes, autour des valeurs de l'égalité, de la solidarité, de la réciprocité, de l'amitié, etc. Le «nouveau partenariat » est toutefois adapté aux normes libreéchangistes de l'OMC (Organisation mondiale du Commerce). Il n'y a plus de « liens préférentiels », la logique concurrence exigeant, selon le Préambule de l'Accord de partenariat entre les ACP (Afrique, Caraïbes et Pacifique) et la Communauté européenne, le respect des «engagements auxquels [les parties] ont souscrit dans le cadre de l'Organisation mondiale du commerce ».

Du point spéculatif, la première relation asymétrique a recoupé l'opposition raison/émotion, idéologie coloniale théorisée par la négritude senghorienne. Le Nègre est racialement émotif, a affirmé Senghor (1964). Aussi élabore-t-il une division métaphysique opposant l'émotion à la raison hellène ou européenne. Ce dualisme ontologique recouvre une division concrète : l'âme nègre n'étant pas portée sur la science et la technique, l'Europe les lui apportera par le biais de l'aide. C'était l'africanisation de la pensée européenne sur la place subalterne de l'Afrique dans le concert des nations.

Dans une telle vision du monde, la question de l'éducation est réduite à la portion congrue, aux acquêts : la culture européenne, la civilisation scientifique et technique étant des "attributs» de l'Europe, il faut modifier la structure psycho-organique héréditaire de l'Africain, sa race, par métissage, afin qu'il puisse accéder à la modernité scientifique et technique. C'est la théorie de la biologisation du culturel, c'est-à-dire l'idée que la culture est liée à la biologie de chaque groupe racial ou humain. Elle voit par conséquent dans le métissage (biologique, et donc culturel) le moyen d'entrer dans la culture européenne fondée sur la science et la technique. Cette métaphore du métissage est cependant inadéquate pour exprimer les changements qu'exigent nos sociétés. Le processus de reprise de l'initiative historique, la réorganisation du chaos 
culturel créé par la domination, l'emprunt à l'autre lui-même des éléments qui s'avèrent nécessaires pour notre maîtrise de la civilisation industrielle, tout cela est obscurci par la métaphore du métissage culturel ou biologique. Avec une telle métaphore, on confond ordre culturel et ordre biologique dont les rythmes d'évolution sont diamétralement opposés. C'est un truisme que les hommes changent de culture sans changer d'espèce.

La crise idéologique de cette vision du monde amena les Africains à rejeter la citoyenneté d'Empire. En effet, sous le colonialisme, le colonisé n'est pas un sujet politique, l'ordre juridique et politique distinguant civilisés et barbares, citoyens et non-citoyens, vrais et sous-hommes. La double citoyenneté faisait appel à un double collège électoral. Grâce aux valeurs véhiculées par l'école (droit des peuples à disposer d'eux-mêmes, droit de résistance à l'oppression), les Africains montrèrent le caractère contradictoire de la double nationalité : ils retournaient ainsi contre l'État colonial les valeurs démocratiques dont il se réclamait en métropole mais n'appliquait pas en colonie.

Contre les peuples désireux d'accéder aux valeurs universelles de liberté et d'égalité, l'Europe du Traité de Rome a noué alliance et complicité avec des États et des hommes politiques défendant de pseudo-valeurs africaines, notamment l'idée qu'un seul est libre. Cette alliance, au nom de l'amitié, créa une corruption institutionnalisée et la répression de toute liberté d'expression. Les accords de Yaoundé, puis de Lomé, délimiteront le nouveau champ jusqu'aux années 90 : l'Afrique produit des matières premières et l'Europe lui apporte l'assistance technique et scientifique, selon le schéma ontologique élaboré par Senghor, et que nous avons exposé plus haut : il y a d'une part des êtres d'émotion, les Africains, bons pour tout ce qui a trait aux puissances du corps. L'émotion, c'est l'être même du Nègre, son essence ; "Le nègre se définit essentiellement, par sa faculté d'être ému », affirme Senghor (1964, p. 24). A l'émotion s'oppose la raison blanche, principe de la civilisation technicienne comme l'émotion est le principe des civilisations africaines qu'elle a produites et qu'elle va produire : "L'émotion est nègre, comme la raison est hellène » (Senghor, 1964, p. 70). Incapables du point de racial de développer la civilisation technique, les Noirs doivent attendre les productions des Blancs qui la maitrisent, en attendant de se diluer dans la civilisation européenne par métissage biologique et culturel. Senghor théorisait ainsi l'idéologie de l'aide, de la coopération et de l'assistance technique.

En ramenant la culture des peuples noirs à une existence naturelle, instinctuelle, quasi-animale, ils ne peuvent plus la dépasser. C'est une volonté de rendre les rapports sociaux et politiques immuables, alors que l'histoire a montré la transformation rapide des rapports entre les maîtres et les esclaves. Cette philosophie fonda concrètement l'association de l'Afrique et de l'Europe. On constata alors que l'harmonisation des politiques financière, économique et diplomatique entre l'Europe et l'Afrique, conduisit à la persécution de tous les mouvements et individus critiques. L'opposition politique fut interdite, toute 
revendication sociale et syndicale bannie et endiguée au nom du « développement », toute contestation contenue. En un mot, les pays dépendants de l'aide s'inscrivaient alors dans une pente inexorable de la corruption et de sa défense par la répression. Des pouvoirs personnels s'édifient au nom de la tradition et du nationalisme pour enlever tout discours crédible à l'opposition acculée à la clandestinité ou à l'exil, si elle n'est pas décimée.

\section{Eurafrique asymétrique : Partenariat éthico-politique ou d'ajustement économique et culturel ?}

Cette association entre l'Afrique et l'Europe a pris un autre sens avec la fin de la bipolarité et le processus de l'universalité des échanges, c'est-à-dire la nouvelle forme du «marché universel». L'Europe propose désormais à l'Afrique un «principe spirituel» fondamental que l'on peut ainsi énoncer: un ordre pacifique mondial se construit et se réalise au moyen du «régime du Droit» (Art. 2, Accord de partenariat de Cotonou, 2000) et du commerce. Les trois concepts qui le structurent, la Paix, le Droit, le Commerce, s'inscrivent dans l'optique ouverte par les Lumières, notamment le projet d'Emmanuel Kant d'un «droit cosmopolitique ». C'est un ordre éthique, politique et juridique postnational qui doit instaurer une " paix perpétuelle » entre les nations au moyen du caractère pacifique des démocraties, la socialisation par le commerce, la fonction critique de l'espace public. Mais un tel ordre n'est possible que si l'Afrique s'impose des «conditionnalités » d'ordre éthico-politique, notamment l'universalisation de la démocratie, des droits de l'homme, de la bonne gouvernance et de la transparence, la performance dans la gestion, l'autonomisation des acteurs économiques.

Le «nouveau partenariat» s'inscrit dans la logique de l'ajustement structurel. Les élites doivent «s'approprier» son idéologie de la libre-entreprise et la réaliser. L'ajustement économique appelle lui-même un ajustement culturel, métaphysique comme "principe d'appropriation» (ownership) (Accord de Cotonou, art. 11, al. 1; art. 12, al. 47) intime, psychologique des normes de l'inégalité et de l'exclusion. Il s'agit essentiellement de mettre en place le processus de l'accumulation primitive, processus de "l'accumulation du capital » (art. 8, al. 22 ; art. 15, al. 65) absent lors des indépendances. On saisit mieux le ton d'exubérance libérale du texte africain sur « le Nouveau partenariat pour le développement», texte intitulé New Partnership for Africa's Development (NEPAD), et publié en 2001, un an après l' accord de partenariat entre l'Afrique et l'Europe Il s'agit désormais, dit le texte africain, de participer à « une révolution économique mondiale » qui exige que l'Afrique soit polarisée « en classes sociales antagonistes », notamment « une classe animée d'un esprit d'entreprise », " une classe moyenne dotée de compétences et capacités de gestion » (art. 8, al. 21) et des "professionnels qualifiés» (art. 8, al. 22). Le texte regrette un héritage déficient au moment des indépendances, à savoir « une faible classe capitaliste » à cause «d'un faible processus d'accumulation » (art. 
8, al. 22). Ce sont ces faiblesses dans «l'accumulation du capital» (art. 15, al. 65) que le NEPAD (New Partnership for African Development) veut combler au moyen d'une «appropriation» de l'ajustement structurel (art. 12, al ; 47). Le texte partenarial africain reprend le langage cru du darwinisme social : accepter la «mobilité accrue du capital public et privé » qui exige de « rivaliser les uns avec les autres pour trouver des capitaux sur des marchés mondiaux » (art. 9, al. $30)$.

La philosophie partenariale est donc la nouvelle idéologie destinée à l'Afrique. Elle élabore un discours conceptuel de légitimation dont le but est d'émousser l'esprit revendicatif des Africains, en les accusant eux-mêmes et leurs cultures d'être fautives dans les difficultés actuelles de l'Afrique. Cette philosophie partenariale s'exprime dans le courant du postcolonialisme. Non content de falsifier les causes historiques et macro-économiques des maux de l'Afrique, il fonde le "nouveau partenariat» asymétrique sur une dimension ontologique, l'opposition du corps et de l'esprit, socle de l'afro-pessimisme et de l'afrodénigrement. L'opposition du corps et de l'esprit se voit dans la métaphore de la « politique du ventre ». Son pessimisme historique et culturel est hanté par l'idée d'une "tragédie de la culture» africaine. Pour sortir d'une culture autoétrangleuse de toute vie éthique, démocratique et productive et inscrire l'Afrique dans l'économie-monde, il s'agit donc pour le discours postcolonialiste, d'aggraver la surexploitation des dominés africains. Dans cette volonté de mettre en place l'accumulation primitive apparaît une étrange convergence discursive et temporelle entre des traités internationaux et un discours théorique.

Les principes du nouveau partenariat sont déterminés par les normes ultralibérales qui ébranlent le politique, le statut civique des individus. D'abord la légitimité n'est pas, dans les sociétés africaines, le fait du souverain, à savoir le peuple (ou ses représentants) auquel le pouvoir appartient dans un système démocratique; elle est octroyée par l'institution de la créance. Il y a là usurpation et inversion de la légitimité politique : les pouvoirs locaux, tenant leur force financière et économique de l'extérieur, estiment secondaire la recherche de la légitimité sociale et politique. Ensuite les normes libérales, en exigeant un État minimum, saccagent l'école, la santé, le travail de socialisation ; on observe la disparition entre l'interdit et le permis. Rien n'ayant plus de valeur, l'anomie s'installe. Ce que montrent justice populaire, exécutions extrajudiciaires, guerres, violence, pogroms, génocide.

En inscrivant l'ordre marchand au cœur de toute institution, l'ajustement à la mondialisation sape les fondements de l'être en commun, brise les fragiles équilibres des liens sociaux: on revient a des formes ethno-régionales de communauté. Une citoyenneté locale et exclusive des tiers connaît un renouveau dans l'espace public. L'ajustement structurel enserre l'Afrique autour d'une alternative hégémonique, non négociable, sans issue, funeste et mortifère: s'ajuster ou périr. Celle-ci ethnicise et stérilise l'ordre politique; ses «fruits empoisonnés » se voient dans le génocide rwandais et la nouvelle rhétorique de 
l'autochtonie (ivoirité, congolité, gabonité, zambianité, par exemple). Ceux qui viennent les migrants nationaux qui viennent d'autres régions - ou d'ailleurs sont stigmatisés comme des allogènes ou des porteurs d'une «nationalité douteuse », « vagabonde » ou « de circonstance ».

\section{Nouveau partenariat et exclusion}

Deux choses sont donc au fond du «nouveau partenariat ». D'une part, inscrire l'Afrique de façon passive dans l'économie mondiale de la libre entreprise et de la libéralisation des échanges. Aussi faut-il bouleverser les significations sociales et imaginaires pour que les individus acceptent la logique de l'accumulation du capital et de l'exploitation économique. D'autre part, faire accepter la «réadmission des illégaux »: ce principe institue le refus de la mobilité de la force du travail, la nouvelle forme de l'accumulation primitive ayant décrété la « fin du travail».

L'Europe restreignant le droit de circulation, l'article 13 de l'Accord de Cotonou qui porte sur les migrations est un des plus longs, avec cinq alinéas et plusieurs attendus. Prenons la question de l'éducation : le but de l'Europe est désormais d'éviter l'émigration des étudiants africains en leur permettant dans leurs " pays d'origine », un « accès à l'enseignement (...) notamment par l'utilisation des nouvelles technologies de la communication $»$. Cette mesure est discriminatoire. D'une part, dans certains pays européens, des instructions sont données aux postes diplomatiques, aux services préfectoraux et aux universités : ils doivent sélectionner en fonction de l'origine les étudiants pour faciliter la venue d'étudiants dits "prioritaires» ou d'attirer les meilleurs. Parmi ceux-ci ne figurent pas les étudiants africains. Ensuite, l'Europe favorise l'immigration des catégories professionnelles hautement qualifiées. Dans tous les cas, les programmes éducatifs européens font appel aux étudiants d'autres nationalités jugées économiquement porteuses - «prioritaires » - comme le Japon, la Chine, etc., sans lien institutionnel, culturel, historique ou linguistique avec l'Europe. D'un côté, mobilité réelle avec expériences culturelles vivantes, de l'autre, mobilité virtuelle. L'irénisme lyrique des philosophies afro-mondialistes et de la «traversée » voit ici sa limite: le discours sur le besoin de «s'ouvrir à l'altérité » parce que le monde n'est plus une menace pour les Africains, mais un "vaste réseau d'affinités» se heurte au principe de réalité de la politique migratoire européenne. Comment en effet « traverser des expériences », s'il n’y pas déplacement, rencontre, interaction, selon le vœu kantien.

Contre l'irénisme postcolonialiste, le monde reste celui de la lutte, du conflit, des rapports de force. Et ce, tant qu'il n'y aura pas entre les nations des niveaux de puissances matérielles équivalents. Notre maîtrise de la civilisation industrielle ne se fera pas autour d'un verre, avec des tapes amicales au dos; ce sera une conquête, un arrachement. Enfin, l'introduction des technologies de la communication comme mode privilégié d'éducation suppose une triple 
révolution culturelle : conduire une alphabétisation systématique, introduire le téléphone dans les moindres bourgades, électrifier partout. Les conventionnels européens et africains en sont loin, et surtout loin de la pensée prospective d'un Rajiv Ghandi qui, pour donner corps à la révolution informatique en Inde, a électrifié tout le pays afin d'installer des ordinateurs dans toutes les écoles.

La logique partenariale est plutôt défensive: elle tourne autour de «la réadmission des immigrants illégaux » qui participe de l'arsenal européen de « maitrise des flux migratoires ». Il s'agit en somme d'interrompre la fuite des exclus, de stabiliser, de contrôler et d'encadrer les mouvements de la force de travail et du salariat en érigeant les barrières à sa mobilité. Quittant les systèmes traditionnels de dépendance, ces exclus n'accèdent pas pour autant à un statut civique protégé. Au lieu de quoi les Africains sont culpabilisés : on les accuse d'être «victimes d'eux-mêmes » en refusant l'exploitation; ils s'autoexcluent de la modernité industrielle à cause de leur incapacité à s'autogouverner, à cause de leur incapacité économique. Ils doivent donc s'en prendre à eux-mêmes.

\section{Vous avez dit citoyenneté ?}

Cette situation est contemporaine du dessein européen : il s'agit que l'Europe devienne une puissance mondiale dans le cadre de la mondialisation et de la redistribution de l'hégémonie dans l'espace public international. D'où des mesures d'austérité économique à l'intérieur, avec la définition, à partir de Maastricht en 1991, d'une citoyenneté européenne désormais ajoutée à la nationalité de chaque État membre (libre circulation et de séjour, pouvoir européen avec pouvoir de codécision, droit d'éligibilité aux élections municipales et parlementaires européennes, droit de protection consulaire par tout autre État européen, droit de pétition et de plainte).

Cette citoyenneté est toutefois étagée et exclusive d'extracommunautaires, souvent citoyens de seconde zone au statut précarisé au gré des aléas économiques et politiques. À l'extérieur, l'Europe restreint le droit de circulation pour se préserver de la subversion, de toute invasion, voire de la perte de l'équilibre ethnique. Le partenariat est donc au service de la maîtrise des flux migratoires. La peur de l'immigration est la seule politique - défensive et policière - à l'égard de l'Afrique. Le monde africain, rangé au rang des barbares indésirables, doit être contenu à l'extérieur des frontières. Mais l'extérieur est purement formel, l'indésirable ayant la figure du banlieusard d'origine africaine dont les «incivilités » font les unes. Il devient l'ennemi intérieur à éradiquer. A son propos, Balibar voit persister le néocolonialisme autant dans la «façon dont la police s'y comporte, que dans les expéditions militaires françaises au Congo-Brazzaville et en Côte-d'Ivoire » (Balibar, 2003, pp. 166-167). 
Une autre explication est donnée à l'identité de traitement de l'Africain dans l'Hexagone et en Afrique. Pour Jean-Loup Amselle, la «représentation des Africains » se façonne dans "cette Afrique hexagonale et menaçante pour la République » (2002, p. 59). Dès lors, dans la littérature, l'Africain devient la figure animalisée de l'ennemi qu'une politique eugéniste doit neutraliser et éradiquer. Ainsi, dans son roman Les Particules élémentaires, l'écrivain Michel Houellebecq décrit-il l'Africain des banlieues comme un "Sauvage» qui abaisse le niveau culturel de la civilisation occidentale : "Nous envions et admirons les nègres parce que nous souhaitons à leur exemple redevenir des animaux, des animaux dotés d'une grosse bite et d'un tout petit cerveau reptilien, annexe de leur bite » (Houellebecq, 2001, p. 195). Ce discours relaie les discours publics sur les "sauvageons" ou sur les "nouveaux barbares" élevés à la dimension d'une Menace pour l'ordre républicain et démocratique. L'Africain, indésirable, doit donc être rejeté et sa barbarie contenue par la police ou la canonnière : "Comment ne pas voir dans cette nouvelle vision ethnique des « classes dangereuses », vision assortie de toutes les formes de surveillance et de pistage en continu (vidéo, brigades anti-criminalité) dont dispose l'État la forme ultime de la mondialisation de l'Afrique. Comment ne pas voir dans le couvre-feu pour les mineurs le sommet d'un appareil répressif destiné à dompter une libido reptilienne venue du fond des âges » (Amselle, 2002, p. 60).

Historiquement, pour construire leur identité, les peuples indo-européens ou sémitiques ont dépeint le serpent comme le mal à neutraliser. La métaphore ophidienne de Michel Houellebecq (« un tout petit cerveau reptilien ») et celle de Jean-Loup Amselle ( « une libido reptilienne venue du fonds des âges ») prend ici une dimension existentielle que le philosophe allemand Schelling donnait déjà à l'Orouboros, le serpent tortueux. L'immigré africain, indésirable, dangereux et menaçant, prend la position de l'ennemi intérieur qui apporte l'insécurité et insécurise. Mais cette image est construite artificiellement par l'Europe maastrichtienne : sa quête d'identité depuis le Traité de Rome n'a pas encore abouti de façon positive en dehors d'une zone de libre-échange et l'après 89. Le résultat d'ensemble, c'est la réduction des migrants au statut de population inférieure et soumise aux contrôles policiers, en somme un statut de non-citoyens. Ce régime de discriminations politiques est la face sombre et l'un des obstacles à l'émergence de la citoyenneté européenne transnationale ou postnationale. Les pratiques sécuritaires qu'il suscite désarticulent et annulent la citoyenneté existante et acquise.

\section{Éducation citoyenne comme contrepoids civique et politique}

Pour conclure, aucun partenariat ne peut être crédible sur la base de la peur de l'autre, généralement animalisé (la métaphore du serpent dangereux) ou projeté de façon fantasmatique à travers le schème raciste de la division de l'humanité en barbares et civilisés, sous-hommes et surhommes. Pour être crédible le partenariat entre l'Europe et l'Afrique devrait sortir du contrat pour la loi : il faut 
faire appel au peuple pour légiférer. Le contrat valorise l'ordre marchand et exclut tout intérêt sans lien avec la marchandise. La logique partenariale doit reprendre l'idée kantienne du commerce comme rencontre, interaction, circulation. Il faut donc limiter le pouvoir du marché en donnant toute leur force aux corps intermédiaires dans l'éducation civique et politique pour créer des contrepouvoirs qui établissent la pluralité conflictuelle du social.

À long terme, une proposition énonce un possible qui mobilise la conscience africaine depuis le 19e siècle: tout partenariat où le rapport de forces intellectuelle et pratique est défavorable à l'Afrique doit être aboli. Constituée en centre d'auto-assomption et d'affirmation, repliée sur elle-même et ses besoins réels, l'Afrique consolidera son rassemblement et la démocratie, maîtrisera la civilisation industrielle pour produire elle-même ses biens et services de base. Elle pourra alors s'intégrer activement à l'économie mondiale constituée d'autres centres à la fois repliés sur eux-mêmes et ouverts. Ce n'est que dans un tel cadre qu'elle négociera dignement avec ses partenaires.

L'éducation et la formation des hommes acquièrent ici une importance décisive, la démocratie et la civilisation industrielle supposant une universalisation de l'alphabétisation, une égalisation des conditions. En effet le mode de travail industrielle repose sur la science et la technique, le savoir, la participation consciente à la production. Le débat démocratique y repose sur l'engagement actif d'un peuple éduqué qui reconnaît son droit à la personnalité et qui s'exprime par l'entremise du vote et par sa participation à une société civile critique. En donnant la parole au particulier, ce dernier donne place à la pensée pour soi, la délibération, le débat et l'agir selon des principes déterminants et constituants de la justice et de la liberté.

\section{Notes bibliographiques}

Accord de partenariat ACP-CE, signé à Cotonou, le 23 juin 2000, Le Courrier ACP-UE, septembre 2000, édition spéciale, publiée par La Direction générale du Développement, Commission Européenne, Bruxelles.

Amselle, J. (2002). L'Afrique : un parc à thèmes. Les Temps modernes, n 620-621.

Balibar, É. (2003). L’Europe, l'Amérique, la guerre. Réflexions sur la médiation européenne. Paris : La Découverte.

Balibar, É. (2002). Droit de cité. Paris : PUF.

Braeckman, C. (2003). Les nouveaux prédateurs. Politique des puissances en Afrique centrale. Paris : Fayard.

Chossudovsky, M. (1994). Les fruits empoisonnés de l'ajustement structurel. Le Monde Diplomatique, novembre.

Diop, C. A. (1960). Les fondements économiques et culturels d'un Etat fédéral d'Afrique noire. Paris : Présence Africaine.

Houellebecq, M. (2001). Les Particules élémentaires. Paris : J'ai lu.

Jalée, P. (1973). Le Pillage du tiers monde. Paris : Maspéro.

New Partnership for Africa's Development (NEPAD) (2001). Abuja : Interlink Print.

88 Canadian and International Education Vol. 34 no. 1 - June 2005 
Schelling, F.-W.-J. (1994). Philosophie de la mythologie. Grenoble : Jérôme Millon. Senghor, L. S. (1964). Liberté I. Paris : Seuil.

Towa, M. (1969). Léopold Sédar Senghor, négritude ou servitude ? Yaoundé, Clé, 1969.

Towa, M. (1976). Identité et transcendance. Examen d'un dilemme de la pensée africaine moderne. Thèse de Doctorat d'Etat, Université de Nanterre. 